\title{
A Cross-Layer User Centric Vertical Handover Decision Approach Based on MIH Local Triggers
}

\author{
Maaz Rehan ${ }^{1}$, Muhammad Yousaf ${ }^{2}$, Amir Qayyum $^{1}$, and Shahzad Malik ${ }^{3}$ \\ ${ }^{1}$ CoReNeT, M. A. Jinnah University, Islamabad \\ ${ }^{2}$ Center for Advanced Studies in Engineering (C@SE), Islamabad \\ ${ }^{3}$ COMSATS Institute of Information Technology (CIIT), Islamabad \\ maazrehan@yahoo.com, myousaf@ymail.com, aqayyum@ieee.org, \\ smalik@comsats.edu.pk
}

\begin{abstract}
Vertical handover decision algorithm that is based on user preferences and coupled with Media Independent Handover (MIH) local triggers have not been explored much in the literature. We have developed a comprehensive cross-layer solution, called Vertical Handover Decision (VHOD) approach, which consists of three parts viz. mechanism for collecting and storing user preferences, Vertical Handover Decision (VHOD) algorithm and the MIH Function (MIHF). MIHF triggers the VHOD algorithm which operates on user preferences to issue handover commands to mobility management protocol. VHOD algorithm is an MIH User and therefore needs to subscribe events and configure thresholds for receiving triggers from MIHF. In this regard, we have performed experiments in WLAN to suggest thresholds for Link Going Down trigger. We have also critically evaluated the handover decision process, proposed Just-in-time interface activation technique, compared our proposed approach with prominent user centric approaches and analyzed our approach from different aspects.
\end{abstract}

Keywords: MIH, Vertical Handover Decision, Cross-layer, User Centric Approach.

\section{Introduction}

The process of migration of connection from one type of network to another involves decisions and multiple information as input to the handover decision phase. This information pertains to different layers. For example information like received signal strength, packet error rate, missed beacons, link speed, etc. can only be taken from MAC Layer; connection characteristics like achieved throughput, delay, jitter, etc. can only be taken from Transport Layer; and commercial networks subscription details and user preferences regarding network selection are application layer features. While moving, whenever a Mobile Node (MN) is in overlapping region, effective network selection becomes a task that requires intelligent decision making based upon selected information from multiple layers and therefore it openly speaks off the necessity of a cross-layer design [5]. 
Users would like to avoid inappropriate handover decisions due to varying cost of different wireless access networks. The user may require from the system to choose either a cost effective network, or a best performance network even if it is costly because the connectivity is more important, or the user needs a dynamic hybrid approach which exhibits different behavior in different situations.

802 family of IEEE includes a variety of wireless technologies like 802.11, 802.15, 802.16 that help to establish Local, Personal and Metropolitan area networks respectively. Similarly, cellular networks like GPRS, EDGE, UMTS provide IP support and allow devices to be connected to Wide Area Network. This builds an overall picture in which networks with wide coverage encompass networks with small coverage thus creating overlapping regions and the need of handover. When a Mobile Node (MN) leaves its current network and enters into a new network, a Handover (HO) process is required so that the current end-to-end services of $\mathrm{MN}$ may continue. Horizontal Handover (HHO) happens when MN moves into same network technology. Otherwise it is Vertical Handover (VHO).

Link Layer (L2) notifications help to speed up the process of HO. Abstract or Unified L2 notifications [19, 21, 22, 24] facilitate upper layers to receive these notification in an implementation/link technology independent way. Some of these abstractions have been specifically designed for L3 handover [21, 22], while others are for L3 \& above in general [19, 24]. Test-bed implementations [21, 23] are also available that use Link Up and Link Down triggers to facilitate L2/L3 handover. Our crosslayer solution focuses on handover decision making in a user centric way that intelligently selects a target network among the candidates. After HO decision phase, any mobility management protocol (e.g. MIPv6[21, 22, 23], EMF[25], TCP-migrates[26] etc.) can be used for handover.

Media Independent Handover (MIH) [19] is a proposed framework of IEEE 802.21 WG which provides a generic interface between 'L3 \& above' and 'L2 \& below' for different network technologies, e.g. 802 family, 3GPP and 3GPP2. MIH divides the handover into Initiation, Preparation and Execution phases [17]. Handover execution is the phase in which mobility management protocols execute and $\mathrm{MIH}$ has nothing to do with it. Handover Initiation and Preparation are the phases where MIH is involved. Handover is initiated when observed link layer parameter, e.g. RSS, missed beacons, packet error rate, etc. degrade enough to indicate either a connection breakage or network load. As a result, handover preparation phase starts in which information about the neighboring networks is accumulated through the already active interface with the help of Point of Service (PoS) entity of current network, as proposed by MIH. MIH provides the aforementioned services to the MIH User through MIH_SAP and MIH_LINK_SAP. The MIH_LINK_SAP is replaced by media specific SAPs of the underlying interface. Fig. 1 shows MIH communication of MIH User with WLAN through MIH_SAP and 802.11u MSGCF [20].

Rest of the paper is organized as follows. Section 2 arguments on the existing approaches by discussing different aspects, Section 3 presents proposed idea, Section 4 gives analysis of the proposed idea and Section 5 is conclusion. 


\section{Service Continuity, Network Availability and Subscriptions}

For handover, [11] lists vertical handover decision strategies including Traditional (RSS-based), Decision Function-based (DF), User-Centric (UC), Multiple Attribute Decision Making (MADM), Fuzzy Logic and Neural Network (FL/NN) and Context Aware (CA) based. Normally, decision strategies work as follow: network selection module or score function first calculates weights of parameter(s) like RSS (Received Signal Strength), QoS parameters like delay, packet error rate, jitter, bandwidth, throughput, etc. and contextual parameters like battery status, etc. either through Analytic Hierarchy Process (AHP) [2] or take them from configuration files as input by user or take fixed values. Secondly it computes weighted sum of the selected parameters to obtain network score and compare the cumulative value obtained against each network to select the best network. In the following subsections we discuss various aspects of handover to make the whole process transparent with the examples from state-of-the-art techniques.

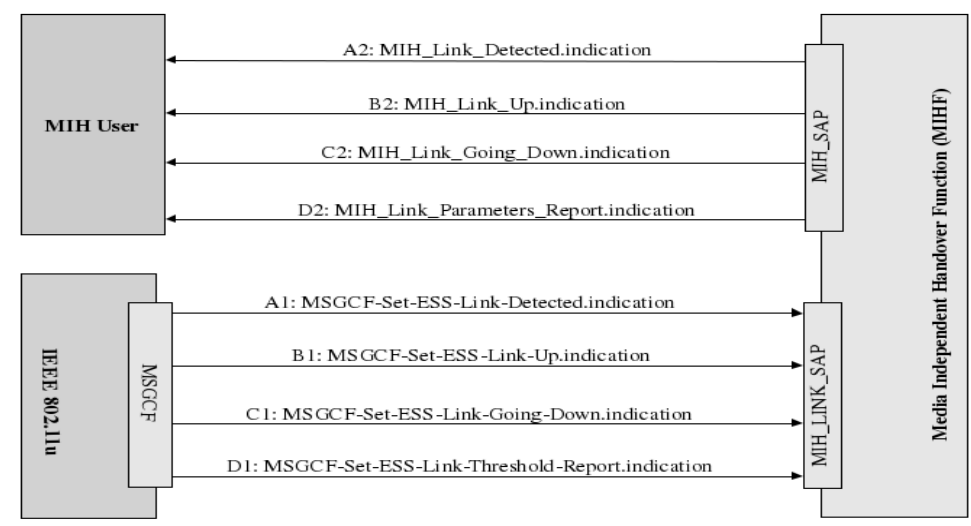

Fig. 1. IEEE 802.21 \& IEEE 802.11u interworking

\subsection{Assignment of Weights, Score Function and Network Selection}

The weighted sum approach $[1,2,3,6,7,8,12,13,14]$ plays a key role in network selection, therefore, inappropriate weight fixing may not bring desired results $[13,14]$. Weights can either be input from user directly [12] or as relative important values (AHP) of QoS parameters. AHP for weight calculation $[1,2,15]$ and ranking is indeed a trusted mechanism but it can prove to be less useful when incorporated in handover scenarios. A mobile user may not have enough knowledge and experience to relate meaningful QoS parameters like jitter, packet error rate, bit error rate, etc in the way they should be related. Since user has to work with relative numbers when adding, deleting or updating a QoS parameter in AHP, the user can undesirably select costly network or pay less cost but with degraded service, because of selecting a network that provides poor services.

Network selection among candidates, based on end-to-end QoS parameters $[1,13,14,15]$ like achieved bandwidth, delay, jitter, throughput, etc., requires a transport 
connection on each interface in order to get access network's QoS parameters. The parameters are then calculated using multi-criteria input function called Score function. The cumulative value is compared with the currently selected network's cumulative QoS value to assess handover requirement. Another mechanism for comparing QoS parameters can be to compare currently selected network's transport parameters with the transport parameters of each candidate network, obtained in the last $\mathrm{N}$ sessions [10], but this approach may not be effective if the network undergoes through frequent changes in a short span of time.

\subsection{Free Access Zone v/s Subscriptions}

When a user moves from his home to office, many small coverage networks (WLANs) may come in his way. MN can connect to any of them, if $i$ ) the network is unprotected, or ii) the $M N$ is an authorized user. It means that $\mathrm{MN}$ can only connect to at most a few of them if above condition is satisfied. For the rest of the time, on the way, it has to connect to broadband or cellular network for service continuity, and therefore, MN needs to be a subscriber of the corresponding wide area network. Practically speaking, most of the subscribers may have a cellular, a braodband and a wireless LAN interface that can adequately fulfill their requirement of service continuity. We also believe that a network's differentiated service to its subscriber is based on the subscribed package, e.g. pre-pay, post-pay etc., and therefore, techniques like network status inquiry or QoS negotiation my be less beneficial for a MN [1].

\subsection{Need for a Network Entity in Handover}

Philosophy of using network entity, e.g. Information Server (IS) is discussed in $[1,8,14]$. When a user carrying multi-homed $\mathrm{MN}$ advances to a vicinity where, there is either a single network or multiple overlapped networks, two possibilities can emerge.

MN does not take 'beforehand QoS information' from IS. In this case MN senses a network when it enters the vicinity. In case of single known network MN will attempt to associate the AP/BS after performing RSS stability check. In case of multiple known networks, it will select the best network based on network pre-subscription information, then perform RSS stability check on the selected network and will keep moving down the list if a network does not pass this check. Finally, it will attempt to associate to the chosen AP/BS.

MN takes 'beforehand QoS information' from IS. In this case, MN first performs IS discovery and then requests for network map i.e. APs/BSs in its way. Now MN can compute best network, well before sensing the beacons, based on network presubscription information. When user enters the vicinity, MN can sensing the signals and perform RSS stability check on the selected network. Rest of the procedure is same as stated above.

This shows that IS benefits in pre-deciding the best network based on subscribed theoretical QoS promises. The disadvantage is its cost to install it in the network which includes IS discovery protocol, a protocol to gather \& maintain network map and a request/response protocol to retrieve network map from IS. 


\section{Proposed VHOD Approach}

Although our proposed approach collects cross-layer context i.e. user preferences and link layer parameters, yet we call it user centric, for, handover is purely based on user preferences. The proposed handover decision policy, Fig. 2, has two major input providers- user preferences and MIHF. As per [19], VHOD algorithm is an MIH User so, it configures link thresholds for receiving link state triggers from MIHF. VHOD algorithm strictly obeys user preferences and executes them when appropriate link state event occurs i.e. when MIHF informs VHOD algorithm about changes in link state through well defined triggers like MIH_Link Detected/Up/Down/Going_Down etc. If handover is required as per user preferences, VHOD issues handover command for mobility management protocol being used in the MN. The proposed handover philosophy is explained below.

\subsection{Cross-Layer Information Gathering}

Cross-layer information gathering comprises of two parts, one is User Preferences gathering while other is Link Layer Information gathering.

Gathering User Preferences. We take network subscription information from user and store them in our cross-layer module. For the user, some networks e.g. Ethernet and $\mathrm{WiFi}$, may be free as he is an authorized user of some local area network (LAN) in a hotel, on the airport, in the office, in the campus or in a conference. Therefore, these

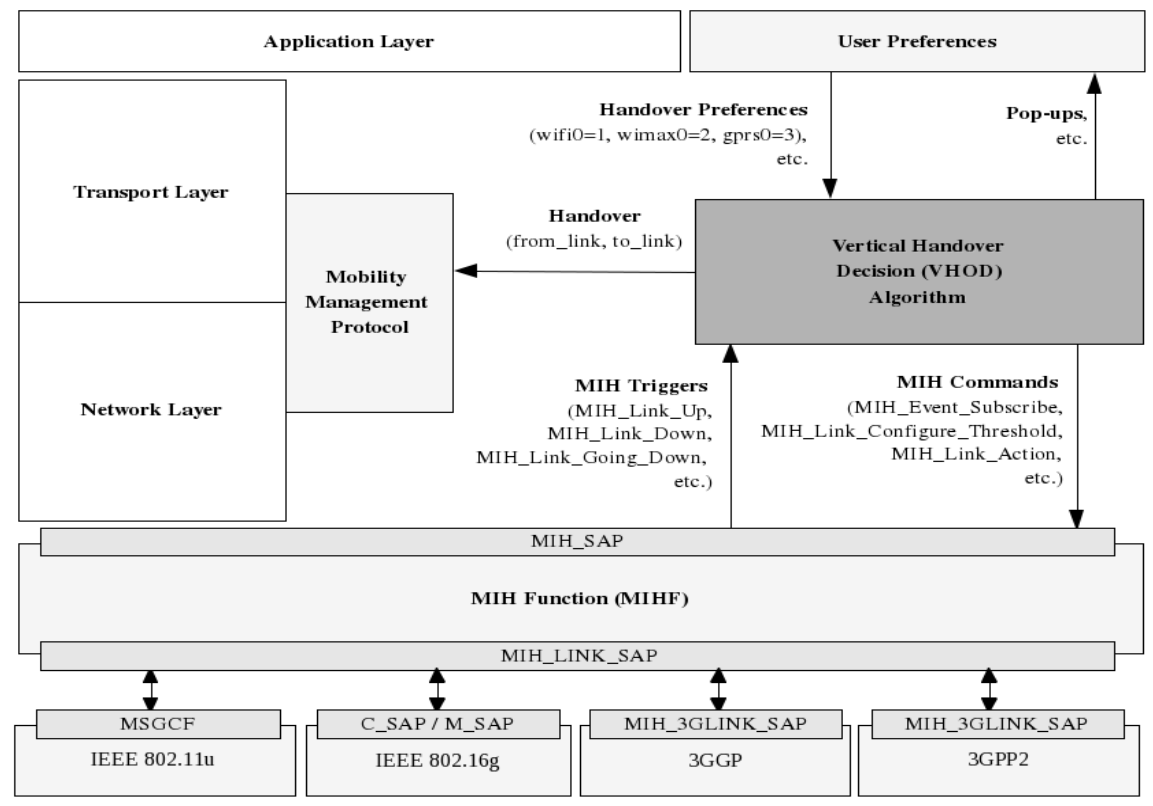

Fig. 2. Block Diagram of Proposed Cross-layer solution for handover management 
networks may always be at the first preference of the user. Finally user is asked to provide his network preferences in descending order for different type of network traffic like Video, Voice and File Download, etc. VHOD will subscribe triggers against those access technologies which are selected by the user as his handover preferences.

User may specify more than one interfaces at same preference e.g. two GPRS connections from two different service providers. In case of links at the same priority we perform scaling of units based on cost. In this way we decide which preference is the best for the user in terms of cost and link utilization.

1. Cost and data rates of both networks (Network-A and Network-B) are in same units, so

DataRate $_{\text {Scaled }}=\left(\right.$ DataRate $_{\text {Network-A }} /$ Cost $\left._{\text {Network-A }}\right) *$ Cost $_{\text {Network-B }}$

2. Data rates are specified in different units, e.g. Net_A in per Mb while Net_B in per Hour, often the case in cellular network. First we have to find megabits per hour for Link_B, and then to perform step 1, described above, to scale the two costs. DataRate $_{\mathrm{Mb} / \mathrm{h}}=$ DataRate $_{\mathrm{b} / \mathrm{s}} * 3600 /(8 * 1024 * 1024)$

Gathering Link Layer Information. At L2, when a threshold set by MIH User is crossed, or an adaptive algorithm running in that layer detects link state change, it notifies MIHF and MIHF in turn notifies MIH User.

Link Detected event is triggered when MN senses beacons from nearby APs and informs upper layers about the details of that AP like MAC address, frequency, networkID, signal strength, SNR, etc. Link Up event is triggered when L2 connectivity of $\mathrm{MN}$ is established with the AP which can be the result of an association and/or an authentication. Link Down is triggered when MN looses L2 connectivity with an AP either because of disassociation or de-authentication. When these happen, current wireless implementations generate a Link Detected/Up/Down trigger and same is specified in [20]. It means threshold for link going down trigger needs to be specified based on scientific grounds.

LGD is generated either by an RSS predictive algorithm running inside SME or by 802.11u MAC due to KEY_EXPIRATION, LOW_POWER, etc [20]. There are two ways to receive LGD trigger. One is to explore a safe LGD threshold value through experimentation. By safe, we mean a threshold value which when meets, application has ample time in seconds or milliseconds, before predicted Link Down, to scan for other networks. Second approach is to write an adaptive program that predicts Link Down, well before time, such that scanning for other networks can be performed within predicted link down time. For WLAN, we adopted the first approach.

We performed following experiment for more than a dozen time, to find LGD threshold. In this regard, we moved with different pedestrian speeds and followed different mobility patterns. We used Fedora 9 platform and ZyXEL P-320W wireless router (AP) and executed each experiment for 5 minutes.

Experiment to find Link Going Down Threshold. Each experiment contained two programs both written in C language. One program captured RSS value after every 5$7 \mathrm{~ms}$ while other recorded timestamped RSS when PING Echo-reply was received. Features of recording time-stamp and RSS were added in source code of PING [18] program available in fedora 9 . PING packets were sent after every 0 milliseconds. 
RSS behaviour was analyzed with different speeds and mobility patterns inside building, 'outside building within AP boundary' and very close to AP boundary (inside/outside). Results of experiments are summarized in Table 1. We conclude that,

1. Safe and adequate RSS capturing interval is every 200th millisecond

2. Near AP boundary, packets drop frequently between $-89 \mathrm{dbm}$ and $-93 \mathrm{dbm}$

3. As soon as RSS lowers to $-94 \mathrm{dbm}$ or $-95 \mathrm{dbm}$, MN looses L2 connectivity with AP, very often, i.e. MN frequently disassociates with AP.

Table 1. Summary of Link Going Down Thresholds

\begin{tabular}{|c|c|c|c|}
\hline Application Type & $\begin{array}{c}\text { LGD } \\
\text { Threshold } \\
\text { (RSS in dbm) }\end{array}$ & $\begin{array}{c}\text { Link Down } \\
\text { Predict Time } \\
\text { (milliseconds) }\end{array}$ & $\begin{array}{c}\text { L2 Connectivity / } \\
\text { Packet Drop }\end{array}$ \\
\hline $\begin{array}{c}\text { Sensitive to Data } \\
\text { Loss }\end{array}$ & $>=-88$ & $\sim 1000$ & Present / Occasional \\
\hline Other & -89 to -93 & $\sim 1000$ to $\sim 0$ & Present / Frequent \\
\cline { 2 - 4 } & $-94 \&-95$ & $<=0$ & Rare / Frequent \\
\hline
\end{tabular}

\subsection{Network Selection Procedure: VHOD Algorithm}

VHOD is based on cross-layer information i.e. the information taken from application layer in the form of user preferences and information taken from $\mathrm{MIH}$ in the form of triggers. When MIHF trigger is received, VHOD algorithm immediately runs strictly following user preferences. Network selection algorithm is depicted in Fig. 3., where we can notice that 'No' procedure is specified against MIH_Link_Detected trigger because the functionality against this trigger is understood, which is, that, $\mathrm{MN}$ will only connect to an APs whose ESSID or any other network ID is specified in configuration file.

\section{Analysis of Proposed Technique}

The parameters like throughput, handover signaling overhead, handover latency, etc. are related to mobility management protocol and out of the scope of this work.

\subsection{Complexity Analysis of VHOD}

Time complexity of our VHOD algorithms is $\mathrm{O}(\mathrm{n})$ for single traffic type. We use linked list (LL) for storing user preferences thus space complexity is CONSTANT. The reason for using LL is that user preferences may change over the time frequently or infrequently and therefore, storing data in 2D static or dynamic array would be more costly than LL in terms of addition, deletion or changing a preference priority. The space complexity of LL in this particular case is CONSTANT because the number of interfaces on MN will be limited, as discussed above. VHOD algorithm performs look-up in the nodes and selects the most suitable from the ordered list after simple comparison. 


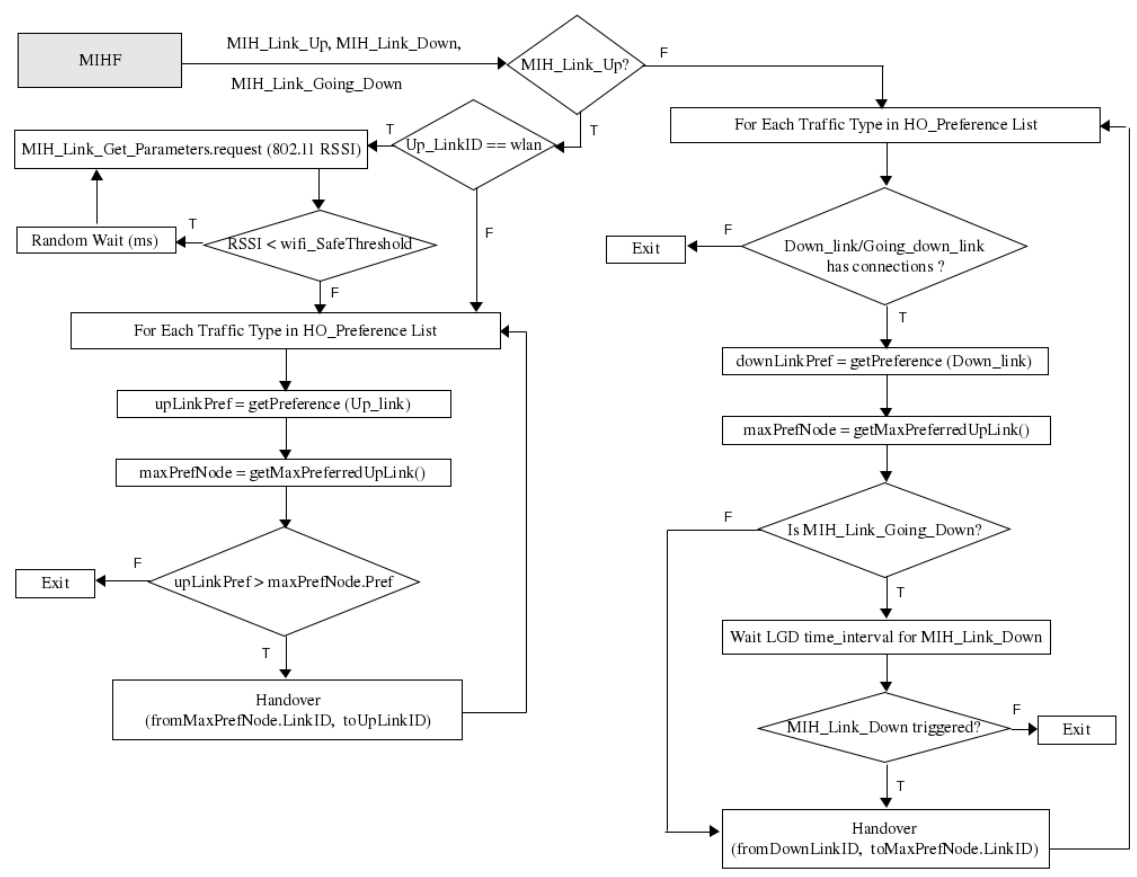

Fig. 3. Proposed Vertical Handover Decision (VHOD) Algorithm

\subsection{Delay in Link Selection}

In our handover approach, link selection delay depends on two factors, viz. i) the time to take handover decision and, ii) the handover decision process. We have shown that complexity of handover decision process is $O(n)$, for single traffic type (audio, video or download). Time to take handover decision is tied with two triggers, i) link down and, ii) link going down. In our case LGD is related to threshold value that we obtained through experimentation. Out of 7 experiments, 5 experiments generated in time HO command which proves lower delay.

\subsection{Interface Activations for Scanning Candidate Links}

Interface activations within a network depends on the dwell time of $\mathrm{MN}$ which in turn depends on the speed of user. We have compared our 'Just-in-time' interface activation technique with 'always on' and 'periodic' interface activation techniques. If each interface activation consumes One Unit of battery, then Just-in-time consumes almost CONSTANT units of battery from $2-20 \mathrm{~km} / \mathrm{h}$ speed while other techniques consume more battery, even at $20 \mathrm{~km} / \mathrm{h}$ speed (see Fig. 4). Just-in-time only suffers when MN's movement is near cell boundary. Here, $\mathrm{MN}$ can also suffer from handover ping-pong effect, but we have remedied it (see Fig. 3). 


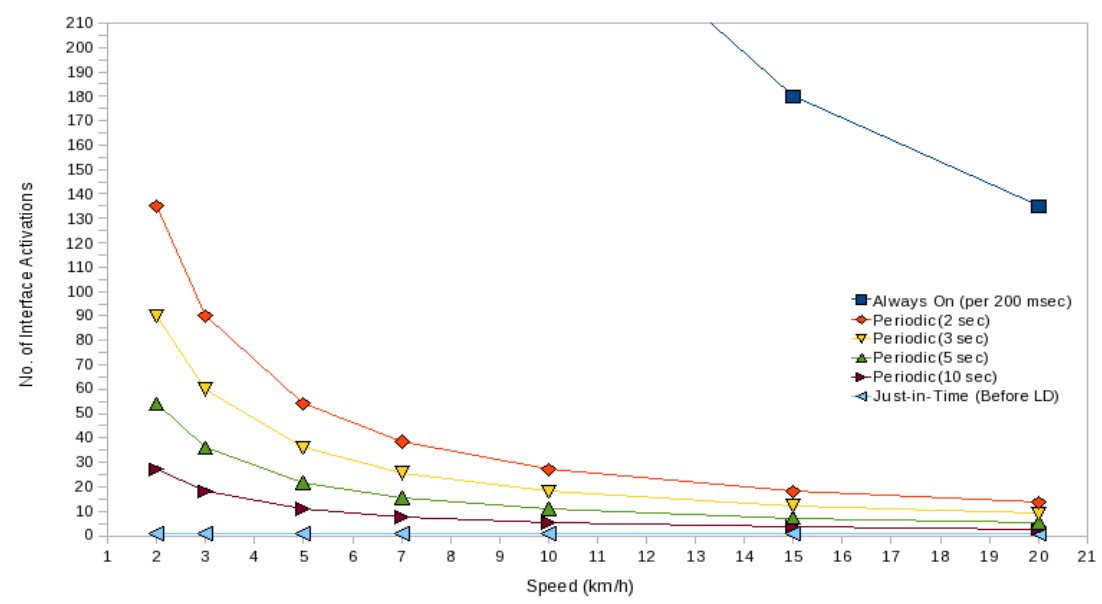

Fig. 4. Comparison of interface activations for different intervals

\subsection{Handover Ping-Pong Effect}

If MN keeps moving near cell boundary, the threshold will be crossed time and again which can lead to "HO to WiMAX" followed by "HO to WLAN", and so on. Once WiMAX is joined then coming back to WLAN, in case of same or different AP, just based on MIH_Link_Up trigger may be short sighted decision, so a stability check is performed before handing traffic over to WLAN from broadband or cellular network. When MN receives MIH_Link_Up event, it requests MIHF for RSSI of 802.11 through MIH_Link_Get_Parameter. If captured RSS is greater than wifiSafeThreshold value $(-86 \mathrm{dbm})$, normal handover process is activated otherwise this process is repeated after short random interval until MIH_Link_Down is received for WLAN (see Fig. 3).

\subsection{Comparison with Other User Centric Techniques}

Table 2 highlights that, the weaknesses of other prominent User Centric techniques are the strengths of VHOD approach e.g. RSS threshold obtained through experiments,

Table 2. Table showing comparison of User Centric HO algorithms with VHOD algorithm

\begin{tabular}{|c|c|c|c|c|c|c|c|c|}
\hline Criteria & {$[1]$} & {$[\mathbf{2}]$} & {$[\mathbf{4}]$} & [8] & {$[\mathbf{9}]$} & {$[\mathbf{1 2}]$} & {$[\mathbf{1 5}]$} & VHOD \\
\hline Provides experimental RSS threshold value & N & N & N & N & N & N & N & Y \\
\hline Generates 'Scan' trigger before Handover & N & N & Y & N & Y & N & N & Y \\
\hline $\begin{array}{l}\text { Requires Transport Connection for getting } \\
\text { Candidate Networks' QoS parameters }\end{array}$ & Y & Y & N & N & N & Y & N & N \\
\hline $\begin{array}{c}\text { Requires Network Entity, e.g. IS to get } \\
\text { Candidate Networks' QoS Parameters }\end{array}$ & N & N & Y & Y & Y & N & Y & N \\
\hline Dwell Timer before handover & Y & N & N & Y & Y & N & Y & Y \\
\hline 'MIH or L2' Trigger Support & N & N & N & N & N & N & N & Y \\
\hline Defines expected link availability time & N & N & N & N & N & N & N & Y \\
\hline
\end{tabular}


generation of a scan trigger well before Handover and mentioning expected link availability time. VHOD selects a network based on the agreed QoS instead of achieved QoS of Candidate Links by establishing a Transport connection or using Information Server while other techniques use one of them. Similarly VHOD takes additional benefit of $\mathrm{MIH} / \mathrm{L} 2$ trigger support.

\section{Conclusion}

Our cross-layer approach for performing vertical handovers based on MIH triggers is new in the domain of User Centric handover approaches. Although MIH has not been widely deployed but the Link Up, Link Down, Link Going Down and Link Get Parameters triggers can be provided through the current Linux or Windows based drivers implementation. VHOD approach works well for QoS and non-QoS applications. Our experimental proof of LGD threshold, per 'traffic type' linear complexity handover algorithm, "Just-in-time" interface activation technique and network selection method based on subscribed QoS makes VHOD approach prominent among the prevailing User Centric approaches.

\section{References}

1. Qingyang, S., Abbas, J.: A quality of service negotiation-based vertical handoff decision scheme in heterogeneous wireless systems. European Journal of Operational Research 191, 1059-1074 (2008)

2. Qingyang, S., Abbas, J.: Network Selection in an Integrated Wireless LAN and UMTS Environment using Mathematical Modeling and Computing Techniques. IEEE Wireless Communications, 42-48 (2005)

3. Anita, S., Dr. Nupur, P.: A Review of Vertical Handoff Decision Algorithm in Heterogeneous Networks. In: Mobility 2007, Singapore (2007)

4. Andrea, C., Giuseppe Di, M.: A User-Centric Analysis of Vertical Handovers. In: 2nd ACM International Workshop on Wireless Mobile Applications and Services on WLAN Hotspots, pp. 137-146 (2004)

5. Vineet, S., Mehul, M.: Cross-layer design: A survey and the road ahead. IEEE Communications Magazine 43(12), 112-119 (2005)

6. Wei, S., Qing-An, Z.: A Novel Decision Strategy of Vertical Handoff in Overlay Wireless Networks. In: Fifth IEEE International Symposium on Network Computing and Applications, NCA 2006 (2006)

7. Paramad, G., Saxena, S.K.: A Dynamic Decision Model for Vertical Handoffs across heterogeneous Wireless Networks. In: PWASET, July 2008, vol. 31 (2008)

8. Christian, M., Samuel, P.: Adaptive handoff scheme for heterogeneous IP wireless networks. Computer Communications 31, 2094-2108 (2008)

9. Wen-Tsuen, C., Yen-Yuan, S.: Active Application Oriented Vertical Handoff in NextGeneration Wireless Networks. In: IEEE Communications Society/WCNC (2005)

10. Olga, O., John, M., Gabriel-Miro, M.: Utility-based intelligent network selection in beyond $3 \mathrm{G}$ systems. In: IEEE International Conference on Communications (ICC 2006), vol. 4, pp. 1831-1836 (2006)

11. Meriem, K., Brigitte, K., Guy, P.: An overview of vertical handover decision strategies in heterogeneous wireless networks. Computer Communications 31, 2607-2620 (2008) 
12. Jiping, L., Yuanchen, M., Satoshi, Y.: Intelligent Seamless Vertical Handoff Algorithm for the next generation wireless networks. In: Mobilware 2008, Innsbruck, Austria, vol. 278, Article No. 23 (2008)

13. Rami, T., Jacques, D., Guy, P.: A Trusted Handoff Decision Algorithm for the Next Generation Wireless Networks. IJCSNS 8(6) (June 2008)

14. Rami, T., Salazar, O., Guy, P.: Vertical Handoff Decision Scheme Using MADM for Wireless Networks. In: WCNC (2008)

15. Shun-Fang, Y., Jung-Shyr, W., Hsu-Hung, H.: A Vertical Media-Independent Handover Decision Algorithm across Wi-Fi and WiMAX Networks. In: 5th IFIP International Conference on Wireless and Optical Communication Networks, Indonesia (2008)

16. IEEE Standard for Local and Metropolitan Area Networks: Part16: Air Interface for Fixed and Mobile Broadband Wireless Access Systems, Amendment 3: Management Plane Procedures and Services, IEEE Std $802.16 \mathrm{~g}^{\mathrm{TM}}-2007$ (2007)

17. IEEE 802.21 official website- 802.21 Tutorial, p. 18 (2007), http: / /www. ieee802.org/21/Tutorials/ $802 \% 2021$-IEEE-Tutorial.ppt (accessed March 09, 2009)

18. Code of PING Command for Fedora 9 platform, http://www.skbuff.net/iputils/iputils-current.tar.bz2 (accessed March 09, 2009)

19. IEEE Standard for Local and Metropolitan Area Networks: Media Independent Handover Services, IEEE 802.21-2008 (January 21, 2009)

20. IEEE Standard for Local and Metropolitan Area Networks: Part11: Wireless LAN Medium Access Control (MAC) and Physical Layer (PHY) specifications

21. Amendment 7: Interworking with External Networks, IEEE p802.11u TM/D5.0 (February 2009)

22. Gogo, K., Shibui, R., Teraoka, F.: An L3-Driven Fast Handover Mechanism in IPv6 Mobility. In: SAINTW 2006, USA, January 23-27 (2006)

23. Teraoka, F., et al.: Unified Layer 2 (L2) Abstractions for Layer 3 (L3)-Driven Fast Handover. RFC 5184 (2008)

24. Montavont, N., Noel, T.: Stronger Interaction between link layer and network layer for an optimized mobility management in heterogeneous IPv6 Networks. Pervasive and Mobile Computing 2(3), 233-261 (2006)

25. Wellens, M.: Enabling Seamless Vertical Handovers Using Unified Link-Layer API. In: Mobility 2006, Bangkok, Thailand, October 25-27 (2006)

26. Yousaf, M., Qayyum, A.: On End-to-End Mobility Management in 4G Heterogeneous Wireless Networks. This paper appears in INCC 2008, Pakistan, May 1-3 (2008)

27. Snoeren, A.C., Balakrishnan, H.: An End-to-End Approach to Host Mobility. In: ACM MOBICOM Conference Boston, USA, pp. 155-166 (2000) 